\title{
ACTIVIDAD ANTIOXIDANTE Y ENZIMÁTICA DE ALBAHACA 'NUFAR' (Ocimum basilicum L.) ALMACENADA EN REFRIGERACIÓN'1
}

\author{
Eduardo López-Blancas ${ }^{2}$,María Teresa Martínez-Damián², María Teresa Colinas-León², \\ Cecilio Bautista-Bañuelos ${ }^{2}$, Juan Martínez-Solís ${ }^{2}$, Juan Enrique Rodríguez-Pérez ${ }^{2}$
}

\begin{abstract}
RESUMEN
Actividad antioxidante y enzimática de albahaca "Nufar" (Ocimum basilicum L.) almacenada en refrigeración. El presente estudio tuvo como objetivo evaluar el efecto del almacenamiento en refrigeración sobre la actividad antioxidante y actividad enzimática en albahaca 'Nufar'. En la Universidad Autónoma Chapingo (Texcoco, México), durante el ciclo primavera-verano de 2012, albahaca previamente empacada en película plástica, se almacenó en cámaras frigoríficas a 5 y $10{ }^{\circ} \mathrm{C}, \mathrm{y}$ a temperatura ambiente $\left(20{ }^{\circ} \mathrm{C}\right.$ - testigo), por dieciocho días. Cada 48 horas, se evaluó el contenido de fenoles totales, capacidad antioxidante y la actividad de las enzimas catalasa, superóxido dismutasa, peroxidasa y polifenol oxidasa. En relación con el tiempo en refrigeración a $5^{\circ} \mathrm{C}$ a los dos y cuatro días se presentó la mayor actividad de catalasa $(14,3 \mathrm{U} / \mathrm{mg} / \mathrm{pro})$ y superóxido dismutasa $(2,9 \mathrm{U} / \mathrm{mg} / \mathrm{pro})$, y a los diez y dieciocho días hubo aumento de la actividad de peroxidasa $(57,6$ y $74,9 \mathrm{U} /$ $\mathrm{mg} / \mathrm{pro}$ ). A $10^{\circ} \mathrm{C}$ y diez días se incrementó el contenido de fenoles totales de 3,7 a $4,2 \mathrm{mg} / \mathrm{kg} / \mathrm{PFr}$; así como la capacidad antioxidante de 47,5 a 79,1 mg VCEAC/g/PFr a ocho días; también aumentó la actividad de la polifenol oxidasa de 11,7 a $31,8 \mathrm{U} / \mathrm{mg} / \mathrm{pro}$ a diez días. El almacenamiento a $5{ }^{\circ} \mathrm{C}$ afectó el contenido de fenoles totales y capacidad antioxidante, así como la actividad enzimática de peroxidasa y polifenol oxidasa, e incrementó la actividad de catalasa y superóxido dismutasa, por lo cual, se sugiere el almacenamiento de albahaca "nufar" a $10{ }^{\circ} \mathrm{C}$ para disminuir la actividad de las enzimas oxidativas.
\end{abstract}

Palabras clave adicionales: pardeamiento, catalasa, superóxido dismutasa, peroxidasa, polifenol oxidasa.

\begin{abstract}
Antioxidant capacity and enzymatical activity of basil 'nufar' (Ocimum basilicum L.) stored under cooling. The present study aimed to evaluate the effect of refrigerated storage on the antioxidant activity (ability to capture free radicals) and enzymatic activity in basil 'Nufar'. This study was conducted at Chapingo University (Texcoco, Mexico) during the spring-summer of 2012. Basil previously packed in plastic film was refrigerated at 5 and $10^{\circ} \mathrm{C}$, and at room temperature $\left(20^{\circ} \mathrm{C}\right.$ - control) for 18 days. Every $48 \mathrm{hrs}$, total phenols, antioxidant capacity and the activity of the enzymes catalase, superoxide dismutase, peroxidase and polyphenol oxidase, were evaluated. Cooling at $5{ }^{\circ} \mathrm{C}$ had the highest catalase activity $(14,3 \mathrm{U} / \mathrm{mg} / \mathrm{pro})$ and superoxide dismutase $(2,9 \mathrm{U} / \mathrm{mg} / \mathrm{pro})$ at 2 and 4 days of storage, while peroxidase activity increased at 10 and 18 days of storage (57,6 and 74,9 $\mathrm{U} / \mathrm{mg} / \mathrm{pro}$ ). At $10{ }^{\circ} \mathrm{C}$ the content of total phenols increased from 3,7 to $4,2 \mathrm{mg} / \mathrm{kg} / \mathrm{WF}$ after 10 days of storage, and the antioxidant capacity from 47.5 to $79.1 \mathrm{mg} \mathrm{VCEAC} / \mathrm{g} / \mathrm{WF}$ after 8 days of storage; the activity of polyphenol oxidase also increased from 11,7 to $31,8 \mathrm{U} / \mathrm{mg} /$ pro after 10 days of storage. The storage at $5{ }^{\circ} \mathrm{C}$ affected the total phenolic content and antioxidant capacity as well as the enzymatic activity of peroxidase and polyphenol oxidase, and increased the activity of catalase and superoxide dismutase, and therefore, in order to reduce the activity of the oxidative enzmes, it is suggested to store basil "nufar" at $10{ }^{\circ} \mathrm{C}$.
\end{abstract}

Keywords: browning, catalase, superoxide dismutase, peroxidase and polyphenol.

\footnotetext{
Recibido: 28 de noviembre, 2013. Aceptado: 30 de junio, 2014. Este trabajo es parte de la tesis en Doctorado en Ciencias en Horticultura, Universidad Autónoma Chapingo, México, del primer autor.

2 Departamento de Fitotecnia, Universidad Autónoma Chapingo. Km. 38.5 Carretera México - Texcoco. Chapingo, Texcoco, Estado de México. C. P. 56230. México. Teléfono: (595)9521616 ext.6163. elob.5.10@gmail.com, teremd13@gmail.com (Autor para correspondencia), lozcol@gmail.com, cecilio_bautistaver@hotmail.com,juanmtz91@hotmail.com, erodriguezx@yahoo.com.mx
} 


\section{INTRODUCCIÓN}

El consumo de frutas y vegetales es de gran importancia en la protección y prevención del envejecimiento, cáncer, enfermedades crónicodegenerativas, causadas por el estrés oxidativo, que origina la liberación de radicales libres de oxígeno (RLO) en el cuerpo; la acción de los RLO es neutralizada por el contenido y capacidad de compuestos antioxidantes mediante la donación de uno de sus propios electrones, protegiendo a las células contra el daño oxidativo (Kaur y Kapoor, 2001; Arcila et al., 2004; Soto et al., 2012).

Los RLO (superóxido: $\mathrm{O}^{-2}$, hidroxilo: $\mathrm{OH}-$, peróxido de hidrógeno: $\mathrm{H}_{2} \mathrm{O}_{2}$ ) son moléculas que reaccionan rápidamente con otros compuestos, tratando de capturar electrones necesarios para ganar estabilidad (Prior y Cao, 2000; Kaur y Kapoor, 2001; Rodríguez et al., 2006).

Generalmente las plantas con alta actividad antioxidante contienen compuestos fenólicos que actúan como agentes reductores, donantes de hidrógeno y extintores del oxígeno singlete (Wang et al., 1996; Connor et al., 2002; Javanmardi et al., 2003). En plantas aromáticas como albahaca (Ocimum basilicum), se ha encontrado la presencia de grupos hidroxilo de los compuestos fenólicos (ácidos fenólicos) (Shahidi et al., 1992), que contribuyen a su fuerte capacidad antioxidante (Zheng y Wang, 2001).

Otro mecanismo de control para prevenir el estrés oxidativo en las células debido a la generación de especies reactivas de oxígeno (ERO) es la acción de enzimas tales como la catalasa (CAT) y superóxido dismutasa (SOD) (Sharma et al., 2008); la CAT convierte al $\mathrm{H}_{2} \mathrm{O}_{2}$ potencialmente peligroso en oxígeno molecular y agua, mientras que la SOD cataliza la conversión de los superóxidos a $\mathrm{H}_{2} \mathrm{O}_{2}$ y $\mathrm{O}_{2}$ (Hammer, 1993; Oueslati et al., 2010).

Las plantas aromáticas sehan estudiado ampliamente debido al interés de proveer sustancias antioxidantes, y evitar efectos nocivos en la salud (Rodríguez et al., 2006). Además, debido a la preocupación de los consumidores sobre la seguridad de los antioxidantes de origen sintético, se ha buscado reemplazarlos por aquellos naturales (Kirca y Arslan, 2008).

Para conservar la frescura y propiedades naturales de estas hierbas se recomienda su almacenamiento en refrigeración (Cantwell y Reid, 2007). En el caso de la albahaca $(O$. basilicum) este no debe ser a temperaturas menores de $5{ }^{\circ} \mathrm{C}$ puesto que presenta daños por frío (Lange y Cameron, 1994; Núñez et al., 2012).

$\mathrm{Al}$ causarse un daño en las paredes y membranas celulares, la oxidación enzimática tiene lugar rápidamente (Pathare et al., 2013). En este contexto, las enzimas polifenoloxidasa y peroxidasa catalizan la oxidación de fenoles a quinonas, las cuales al reaccionar con proteínas y otros compuestos generan colores pardos y reducen las propiedades sensoriales de textura, color y sabor, disminuyendo la calidad nutricional del alimento (Martínez y Muñoz, 2001; Stewart et al., 2001; Ortega et al., 2010). Esto provoca a su vez, un alto porcentaje de pérdida poscosecha en vegetales (Salveit y Morris, 1990; Balois et al., 2008).

El contenido de antioxidantes es un parámetro cada vez más importante con respecto a su funcionalidad en la salud y calidad en hortalizas; por lo cual, es de gran interés evaluar los cambios del contenido antioxidante durante el almacenamiento poscosecha (Kaur y Kapoor, 2001; Ayala et al., 2004). De igual forma, la actividad enzimática ha servido en frutos como indicador de alteraciones metabólicas intrínsecas, manifestadas como desórdenes fisiológicos externos (Pérez et al., 1999). Lo anterior hace conveniente cuantificar la actividad de enzimas como CAT y SOD, las cuales catalizan las reacciones que disminuyen las concentraciones de ERO, que en algunas plantas se incrementan por el frío, durante el almacenamiento (Sala y Lafuente, 2000; Aquino y Mercado, 2004).

El presente estudio tuvo como objetivo evaluar el efecto de la refrigeración sobre la actividad antioxidante y enzimática en albahaca 'Nufar' (O. basilicum).

\section{MATERIALES Y MÉTODOS}

\section{Material vegetal}

El material vegetal fue albahaca 'Nufar', del ciclo primavera-verano de 2012, con calidad de exportación, proporcionado por la empresa Glezte S.P.R. de R.L., localizada en Axochiapan, Morelos. En el mes de abril de 2012, después de cosechar la planta, ésta se pre-enfrió durante 24 horas a $10^{\circ} \mathrm{C}$, posteriormente se empacó $250 \mathrm{~g}$ de albahaca, en bolsas de polietileno de baja densidad de $40 \times 60 \mathrm{~cm}$ con seis perforaciones de $0,5 \mathrm{~cm}$ de diámetro por lado. 


\section{Ubicación del experimento y tratamientos}

El experimento y análisis de variables se realizó durante los meses de abril a agosto de 2012, en el Laboratorio de Usos Múltiples de la Universidad Autónoma Chapingo, ubicada en Texcoco, Estado de México, México. La albahaca empacada se almacenó durante dieciocho días a temperaturas de 5 y $10{ }^{\circ} \mathrm{C}$, en cámaras frigoríficas, y a temperatura ambiente $(20$ ${ }^{\circ} \mathrm{C}$ - tratamiento testigo); cada $48 \mathrm{~h}$, cuatro empaques por tratamiento se transfirieron a un ultracongelador $\left(-20^{\circ} \mathrm{C}\right)$.

\section{Procesamiento de muestras}

Con las muestras congeladas se elaboró polvo de acetona (PAc) (Alia et al., 2005); para lo cual, $25 \mathrm{~g}$ de hojas (sin peciolo) más $50 \mathrm{ml}$ de acetona en congelación $\left(-15^{\circ} \mathrm{C}\right)$, se homogenizaron en licuadora por 25 segundos y se filtró al vacío. Después de homogenizar y filtrar en nueve ocasiones, el extracto de acetona (EAc) de las filtraciones se guardó en refrigeración $\left(4 \pm 2{ }^{\circ} \mathrm{C}\right)$; por otra parte, el PAc se dejó secar a temperatura ambiente $\left(20 \pm 2{ }^{\circ} \mathrm{C}\right)$, después de 15 minutos se pesó y almacenó en un ultracongelador $\left(-20{ }^{\circ} \mathrm{C}\right)$. El peso del PAc se determinó en función de la relación peso fresco de hojas maceradas entre peso del polvo seco.

\section{Variables y diseño experimental}

Las variables de respuesta fueron fenoles totales, capacidad antioxidante, actividad enzimática de catalasa, superóxido dismutasa, peroxidasa y polifenoloxidasa (la primera se determinó a partir del EAc y las siguientes mediante el PAc); los resultados de las variables se reportaron con base en peso fresco. Se aplicó un diseño experimental completamente al azar de un factor (temperaturas de almacenamiento), con tres niveles $\left(5,10\right.$ y $\left.20{ }^{\circ} \mathrm{C}\right)$ y nueve días evaluados $(2,4,6,8,10,12,14,16$ y 18 días); la unidad experimental consistió de un empaque de 250 $\mathrm{g}$ de albahaca, con cuatro repeticiones por tratamiento (cada repetición con cuatro sub-repeticiones) por día de evaluación.

Fenoles totales. Se cuantificaron por el método de Folin y Ciocalteu (Waterman y Mole, 1994). A 0,1 $\mathrm{ml}$ de EAc se adicionó 7,9 $\mathrm{ml}$ de agua desionizada y
$0,5 \mathrm{ml}$ del reactivo de Folin y Ciocalteu, la mezcla se agitó vigorosamente y se agregaron 1,5 ml de solución de carbonato de sodio (20\%); posteriormente se dejó reposar por dos horas en oscuridad; después las muestras se leyeron en absorbancia a $760 \mathrm{~nm}$ en un espectrofotómetro. Por medio de una curva patrón de ácido tánico, los resultados se reportaron en $\mathrm{mg} / \mathrm{kg}$ de peso fresco $(\mathrm{mg} / \mathrm{kg} / \mathrm{PFr})$.

Capacidad antioxidante. Se determinó de acuerdo con el método ABTS [2,2-azinobis (3-etilbenzotiazolin-6-ácido sulfónico)] (Rice et al., 1997) modificada por Ozgen et al. (2006), una solución de ABTS $(7 \mathrm{mM})$ con persulfato de potasio $(2,45 \mathrm{mM})$ se mantuvo en oscuridad durante $24 \mathrm{~h}$ a temperatura ambiente; posteriormente la solución se diluyó con buffer fosfato $(0,1 \mathrm{M}, \mathrm{pH} 7,4)$ hasta obtener un valor en absorbancia de $0,700 \mathrm{~nm} \pm 0,02$ en espectrofotómetro calibrado a $734 \mathrm{~nm}$.

El ensayo de las muestras se realizó con 3,9 ml de solución de ABTS (a $700 \mathrm{~nm}$ ) y $0,1 \mathrm{ml}$ de extracto etanólico de PAc $(0,05 \mathrm{~g}$ de PAc en $5 \mathrm{ml}$ de etanol, homogeneizados con 24 horas de reposo), después de dos horas, la lectura se realizó a $734 \mathrm{~nm}$. La cuantificación se realizó mediante curva de calibración con ácido ascórbico, los valores se reportaron en mg de actividad antioxidante equivalente a vitamina $\mathrm{C} / \mathrm{g}$ de peso fresco (mg VCEAC/g/PFr).

Catalasa (EC. 1.11.1.6; CAT). La CAT se extrajo de $0,05 \mathrm{~g}$ de PAc, este se homogeneizó con $5 \mathrm{ml}$ de Tris- $\mathrm{HCl}(0,1 \mathrm{M}, \mathrm{pH} 8,5)$ que contenía $1 \%$ de polivinilpirrolidona (PVP). La mezcla se centrifugó a $12000 \mathrm{~g}$ por $40 \mathrm{~min}$ a $4{ }^{\circ} \mathrm{C}$, se conservó el sobrenadante. La actividad de CAT se determinó por el método descrito por Blackwell et al. (1990), para lo cual, en una celda de cuarzo, se colocaron $3 \mathrm{ml}$ de amortiguador Tris- $\mathrm{HCl}(10 \mathrm{mM}, \mathrm{pH} 8,5)$ y $0,1 \mathrm{ml}$ de $\mathrm{H}_{2} \mathrm{O}_{2}$ al $88 \%$; la reacción se inició al adicionar $0,1 \mathrm{ml}$ del sobrenadante. Por último, se observó el cambio en absorbancia a $240 \mathrm{~nm}$, tomando lecturas a los $15 \mathrm{y}$ $180 \mathrm{~s}$. La actividad enzimática se reportó como unidad de actividad enzimática/mg de proteína (U/mg/prot), donde una $\mathrm{U}$ es igual a la descomposición de $1 \mu \mathrm{mol} /$ min de $\mathrm{H}_{2} \mathrm{O}_{2}$.

Superóxido dismutasa (EC. 1.15.1.1; SOD). La extracción de esta enzima se realizó de $0,05 \mathrm{~g}$ de PAc, al cual, se le adicionaron $5 \mathrm{ml}$ de Buffer $(0,1 \mathrm{M}, \mathrm{pH}$ $7,8)$ y se homogeneizaron por $20 \mathrm{~s}$; luego la mezcla se centrifugó a $12000 \mathrm{~g}$ por $30 \mathrm{~min}$ a $4{ }^{\circ} \mathrm{C}$ y se reservó el 
sobrenadante. Para el ensayo enzimático se empleó la metodología propuesta por Beyer y Fridovich (1987), en la cual se mezclaron $27 \mathrm{ml}$ de Buffer fosfatos $(0,05$ $\mathrm{M}, \mathrm{pH} 7,8)$, que contenía $27 \mathrm{ml}$ de Buffer $(0,1 \mathrm{mM}$, pH 7,8 + EDTA), $1,5 \mathrm{ml}$ de L-metionina $(30 \mathrm{mg} / \mathrm{ml})$, $1 \mathrm{ml}$ de nitro blue tetrazolium $(1,41 \mathrm{mg} / \mathrm{ml})$ y 0,75 $\mathrm{ml}$ de Triton X-100 al 1\%. A $3 \mathrm{ml}$ de esta mezcla de reacción se adicionaron $0,5 \mathrm{ml}$ del sobrenadante y 0,03 $\mathrm{ml}$ de riboflavina $(4,4 \mathrm{mg} / 100 \mathrm{ml})$; la mezcla se agitó e iluminó por siete minutos con luz fluorescente, y posteriormente las lecturas se hicieron en absorbancia a $560 \mathrm{~nm}$. La actividad enzimática se reportó como unidad de actividad enzimática/mg de proteína $(\mathrm{U} / \mathrm{mg} /$ prot), donde una U de SOD es igual a la cantidad de sobrenadante que foto-inhibe el $50 \%$ de la formación de Nitro Blue Tetrazolium formazan (Giannopolities y Ries, 1977).

El incremento en absorbancia debido a la formación de Nitro Blue Tetrazolium formazan por unidad de tiempo equivale a la velocidad de reacción, y la absorbancia en ausencia o en presencia de varias cantidades de SOD se utilizó para determinar el número de unidades/ml de SOD en la solución (Stauffer, 1989).

Peroxidasa (EC. 1.11.1.7; POD). La POD se extrajo de $0,05 \mathrm{~g}$ de PAc que se homogeneizaron por $10 \mathrm{~s}$, con $5 \mathrm{ml}$ de Tris- $\mathrm{HCl}(0,1 \mathrm{M}, \mathrm{pH} 7,1)$ con $1 \%$ de PVP, la mezcla se centrifugó a 12000 g por 40 min a $4{ }^{\circ} \mathrm{C}$, se conservó el sobrenadante. Se aplicó el método de Flurkey y Jen (1978), se mezclaron 2,45 $\mathrm{ml}$ de amortiguador Tris- $\mathrm{HCl}(0,1 \mathrm{M}, \mathrm{pH} 7,1), 0,25 \mathrm{ml}$ de guayacol $(0,1 \mathrm{M}), 0,1 \mathrm{ml}$ de $\mathrm{H}_{2} \mathrm{O}_{2}$ al $0,25 \%$ y 0,2 $\mathrm{ml}$ del sobrenadante. En esta mezcla de ensayo con un volumen total de $3 \mathrm{ml}$, se determinó el cambio de absorbancia a $470 \mathrm{~nm}$ y las lecturas se realizaron a los 30 y 120 s. La actividad enzimática se reportó como unidad de actividad enzimática/mg de proteína $(\mathrm{U} / \mathrm{mg} /$ prot), donde una $\mathrm{U}$ es igual a la formación de $1 \mu \mathrm{mol}$ de tetraguayacol $/ \mathrm{min}$.

Polifenoloxidasa (EC. 1.14.18.1; PFO). Esta enzima se extrajo con el procedimiento utilizado en la extracción de la POD. La actividad enzimática de la PFO se evaluó mediante el método propuesto por Laminkanra (1995) en el cual se emplearon $3 \mathrm{ml} \mathrm{de}$ catecol $(60 \mathrm{mM})$ disuelto en Buffer Tris- $\mathrm{HCl}(0,1 \mathrm{M}$, pH 7,1) y $0,2 \mathrm{ml}$ del sobrenadante, con esta mezcla se evaluó el cambio de absorbancia a $420 \mathrm{~nm}$, tomando las lecturas a los 10 y $60 \mathrm{~s}$. La actividad enzimática se reportó como unidad de actividad enzimática/mg de proteína $(\mathrm{U} / \mathrm{mg} / \mathrm{prot})$, donde una $\mathrm{U}$ es igual a la formación de $1 \mu \mathrm{mol}$ de o-benzoquinona/min.

La proteína se determinó por el método de Bradford (1976), para lo cual, se homogeneizaron por $10 \mathrm{~s}, 0,05 \mathrm{~g}$ de PAc con $5 \mathrm{ml}$ de Tris- $\mathrm{HCl}(0,1 \mathrm{M}$, pH 7,1) el cual tenía $1 \%$ de PVP. Esta se centrifugó a $12000 \mathrm{~g}$ por $40 \mathrm{~min}$ a $4{ }^{\circ} \mathrm{C}$. Se tomaron $0,2 \mathrm{ml}$ del sobrenadante y se le adicionaron $5 \mathrm{ml}$ de solución Coomassie Blue, se agitó y después de 12 min se registró la absorbancia a $595 \mathrm{~nm}$. La cuantificación se hizo mediante una curva de calibración con albúmina de bovino.

Todas las homogeneizaciones se hicieron en baño de hielo, mediante un homogeneizador de tejidos (IKA-T25) a $12000 \mathrm{~g}$, mientras que las lecturas de las muestras se realizaron utilizando un espectrofotómetro (Genesis-10UV). Todos los ensayos se realizaron a temperatura ambiente del laboratorio $\left(24 \pm 2{ }^{\circ} \mathrm{C}\right)$.

Análisis estadístico. Los datos obtenidos de cada variable por día de evaluación se sometieron a un ANDEVA y prueba de medias de Tukey $(\mathrm{p}=0,05)$ con el paquete de análisis estadístico SAS® (Statistical Analysis System, ver. 9.0) (SAS, 2002).

\section{RESULTADOS Y DISCUSIÓN}

Fenoles totales. De cuatro a diez días de almacenamiento (DDA) el contenido fenólico se incrementó en los tratamientos de refrigeración y no presentaron diferencias estadísticas entre ellos, pero fueron diferentes al de $20^{\circ} \mathrm{C}(\mathrm{p}<0,05)$, el cual presentó los valores más altos después de 4 DDA y durante el almacenamiento. Según Kevers et al. (2007), el contenido de compuestos fenólicos es constante en el almacenamiento, sin embargo en casos como ciruela (Prunus domestica), tomate (Solanum lycopersicum), brócoli (Brassica oleracea italica) o uva negra (lisán) (Vitis vinífera) se ha presentado un breve aumento de los compuestos fenólicos durante unos pocos días. También en raíces de jícama en condiciones de frío se ha reportado el incremento en el contenido de fenoles totales (FET) (Cantwell et al., 2002); asimismo, en fresas (Fragaria vesca) almacenadas a $5{ }^{\circ} \mathrm{C}$ y $10{ }^{\circ} \mathrm{C}$, se observó un incremento continuo en el contenido FET, mientras que a $0{ }^{\circ} \mathrm{C}$ los valores fueron constantes, esto sugiere que la temperatura $\mathrm{y}$ 
tiempo de almacenamiento tuvieron un efecto sobre los compuestos fenólicos (Ayala et al., 2004).

A 12 DDA, el contenido de FET disminuyó en los tratamientos de refrigeración, opuesto al testigo que presentó el mayor valor $(4,9 \mathrm{mg} / \mathrm{kg} / \mathrm{PFr})$; y hubo diferencia estadística significativa $(\mathrm{p}<0,05)$ entre los tratamientos. Al respecto, Kalt (2005) comenta que los niveles de antioxidantes fenólicos parecen ser más sensibles a las condiciones ambientales pre y poscosecha; aunque el contenido de ciertos compuestos fenólicos puede aumentar durante las condiciones adecuadas de almacenamiento. Además entre algunos cultivares de albahaca se ha reportado diferencias en las concentraciones de FET, como en el caso de 'Spice Blue' y 'Gecofure' los cuales presentaron niveles más altos de FET, que 'Limón Sweet Dani' y 'Nufar' (Kwee y Niemeyer, 2011).

Después de los 14 DDA el testigo se eliminó por la presencia de pudriciones y marchitez de las muestras, mientras que en los tratamientos de refrigeración a los 16 y 18 DDA, nuevamente se incrementó el contenido de FET y el tratamiento de $10{ }^{\circ} \mathrm{C}$ presentó los valores más altos, siendo estadísticamente diferente $(\mathrm{p}<0,05)$ al de $5{ }^{\circ} \mathrm{C}$. Lo anterior, probablemente se debió a que en el almacenamiento prolongado los fenoles presentan un estado de oxidación intermedio con mayor actividad captadora de radicales, promoviendo o mejorando la oxidación enzimática (Kaur y Kapoor, 2001). Por lo cual, la preservación del contenido fenólico tiene un gran impacto en la calidad debido a la contribución de los fenoles no sólo en las reacciones de pardeamiento enzimático, sino también sobre el valor nutricional del producto (Kevers et al., 2007).

Capacidad antioxidante. Se incrementó la capacidad antioxidante de los 2 a 8 DDA en los tratamientos de 5 y $10{ }^{\circ} \mathrm{C}$ quienes fueron estadísticamente diferentes $(\mathrm{p}<0,05)$ al de $20{ }^{\circ} \mathrm{C}$, este último con los valores más altos durante todo el almacenamiento (Figura 1). Se ha reportado que la capacidad antioxidante (CAP) y el contenido de FET aumentan cuando la temperatura de almacenamiento es elevada, en este contexto, fresas almacenadas a temperatura de $10{ }^{\circ} \mathrm{C}$ presentaron la más alta actividad antioxidante, que las almacenadas a temperaturas más bajas $\left(0\right.$ o $5{ }^{\circ} \mathrm{C}$ ) (Ayala et al., 2004; Jin et al., 2011). También, en algunos productos como pimiento amarillo (Capsicum annuит), espárragos (Asparagus officinalis) y ciruela (Prunus domestica) se ha reportado un aumento de la CAP cuando se almacenan a temperatura ambiente o en refrigeración; sin embargo, en otros como albaricoque (Prunus armeniaca), espinacas (Spinacia oleracea), plátano (Musa paradisiaca), brócoli (Brassica oleracea italica) y puerros (Allium ampeloprasum), disminuyó durante el almacenamiento (Kevers et al., 2007).

A los 8 y 10 DDA el tratamiento de $5{ }^{\circ} \mathrm{C}$ fue estadísticamente diferente a los otros de 10 y $20^{\circ} \mathrm{C}$, quienes presentaron los mayores valores $(79,9 \mathrm{mg}$ VCEAC/g/PFr), y a los 12 DDA hubo diferencia estadística $(\mathrm{p}<0,05)$ entre los tres tratamientos, siendo el de $20{ }^{\circ} \mathrm{C}$ el de mayor CAP $(87,4 \mathrm{mg} \mathrm{VCEAC} / \mathrm{g} /$ $\mathrm{PFr}$ ). Diversos factores tienen un efecto directo sobre la presencia de antioxidantes individuales, tales como el cultivar, temporada de cosecha, factores genéticos o ambientales (Oboh, 2004), sistemas culturales, temperaturas de almacenamiento (Jin et al., 2011), y la madurez (Ahmed y Hussain, 2009; Ghasemnezhad et al., 2011). Para el caso de O. basilicum, el ciclo de cultivo afectó la CAP de sus aceites esenciales, puesto que estos, obtenidos a partir de cultivos de primavera e invierno mostraron una mayor actividad antioxidante que las cosechadas durante el otoño y verano (Hussain et al., 2008). Además se considera que cada hierba generalmente contiene diferentes compuestos fenólicos, y cada uno de estos posee diferentes cantidades de actividad antioxidante (García et al., 2004; Javanmardi et al., 2003).

De los 10 a 18 DDA hubo una disminución de la CAP en los tratamientos de refrigeración, siendo estadísticamente diferentes $(\mathrm{p}<0,05)$ entre ellos hasta los 14 DDA. Algunos autores mencionan que la alta actividad antioxidante depende de su contenido fenólico total (Ahmed y Hussain, 2009); encontrando correlación directa entre los valores de fenoles y los de VCEAC (Kuskoski et al., 2005). No obstante, estas inferencias deben interpretarse con cautela, ya que se han obtenido usando el método de Folin-Ciocalteu, el cual es ampliamente aceptado, aunque no es muy específico, ya que, los compuestos fenólicos y otros compuestos reductores son cuantificados simultáneamente (Santos y Scalbert, 2000). Esto sugiere que la actividad antioxidante no está limitada a los compuestos fenólicos y puede provenir de la presencia de otros metabolitos secundarios antioxidantes, tales como aceites volátiles, carotenoides, vitaminas (Javanmardi et al., 2003); o 

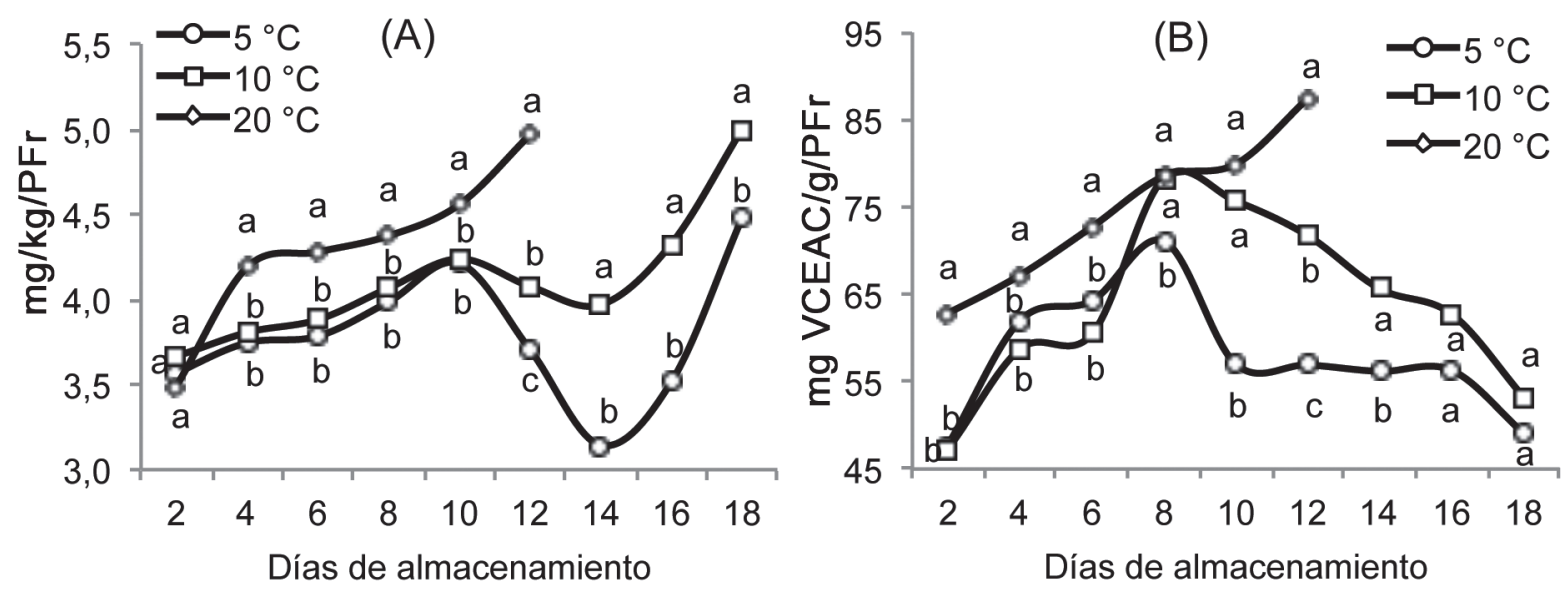

Figura 1. Fenoles totales (A) y capacidad antioxidante (B) en albahaca 'Nufar' almacenada durante dieciocho días a 5, 10 y 20 ${ }^{\circ} \mathrm{C}$. Texcoco, Estado de México, México. 2012.

Medias con igual letra entre los tratamientos de refrigeración en cada día de almacenamiento son estadísticamente iguales de acuerdo con Tukey $(\mathrm{p} \leq 0,05)$.

flavonoides en albahacas verdes y antocianinas en albahacas moradas (Juliani y Simon, 2002).

La capacidad de albahaca como antioxidante natural se ha atribuido a la alta prevalencia de compuestos fenólicos, siendo el ácido rosmarínico, el principal componente activo que se encuentra en $O$. basilicum, y se ha comprobado que tiene valor medicinal y su actividad antioxidante es superior a la vitamina E (R-tocoferol) (Jayasinghe et al., 2003). En $O$. sanctum sus constituyentes interrumpen la cadena de los radicales libres de oxidación mediante la donación de hidrógeno del grupo hidroxilo del fenol, formando de este modo radicales libres estables, evitando así el posterior pardeamiento (Tabassum et al., 2009). Dada la relativa actividad antioxidante de albahaca, se sugiere que esta puede constituir una fuente de compuestos fenólicos antioxidantes en la dieta (Juliani y Simon, 2002).

Catalasa. En lo que respecta a la actividad de catalasa (CAT), a los 4 y 8 DDA, los tres tratamientos fueron estadísticamente diferentes $(p<0,05)$, siendo el tratamiento de $5{ }^{\circ} \mathrm{C}$ que presentó la mayor actividad $(14,3$ y $11,1 \mathrm{U} / \mathrm{mg} /$ prot, respectivamente) en estos días y durante el almacenamiento (Figura 2); este incremento de CAT en el tratamiento de $5{ }^{\circ} \mathrm{C}$ probablemente se debió a que dicha enzima participa en el mecanismo de defensa contra el estrés por frío (Sala y Lafuente, 2000).
A los 6,10 y 12 DDA hubo diferencia $(p<0,05)$ entre los tratamientos de refrigeración con respecto al de $20{ }^{\circ} \mathrm{C}$, el cual obtuvo los menores valores durante el almacenamiento. Inicialmente en los tratamientos de refrigeración se presentó un incremento en la actividad hasta los 4 DDA, con una posterior disminución hasta los 16 DDA; esto concuerda con Trujillo et al. (2006) quienes observaron en flores de 'Duela' (Gerbera jamesonii), un incremento en la actividad de CAT durante los primeros diez días, para posteriormente disminuir hasta el día 30. Por su parte, Sala y Lafuente (2000) determinaron en mandarina (Citrus nobilis) almacenada a $2{ }^{\circ} \mathrm{C}$, una disminución de CAT de 8,1 a 6,5 $\mathrm{U} / \mathrm{mg} /$ prot, después de dos semanas de almacenamiento.

A los 14 DDA hubo diferencia estadística $(p<0,05)$ entre los tratamientos de 5 y $10{ }^{\circ} \mathrm{C}$, los cuales disminuyeron a los 16 DDA e incrementaron a los 18 DDA; este incremento en plantas senescentes, sugiere que la producción en forma natural de altos niveles de radicales libres en tejido senescente, promueve una respuesta antioxidante, incrementando la actividad de CAT (Liang et al., 2003).

Superóxido dismutasa. El estrés ambiental puede inducir una mayor producción de superóxidos dentro de los tejidos de la planta; y para la desintoxicación de las especies reactivas de oxígeno, la planta depende de la enzima superóxido dismutasa (SOD) (Kliebenstein et al., 1998). 

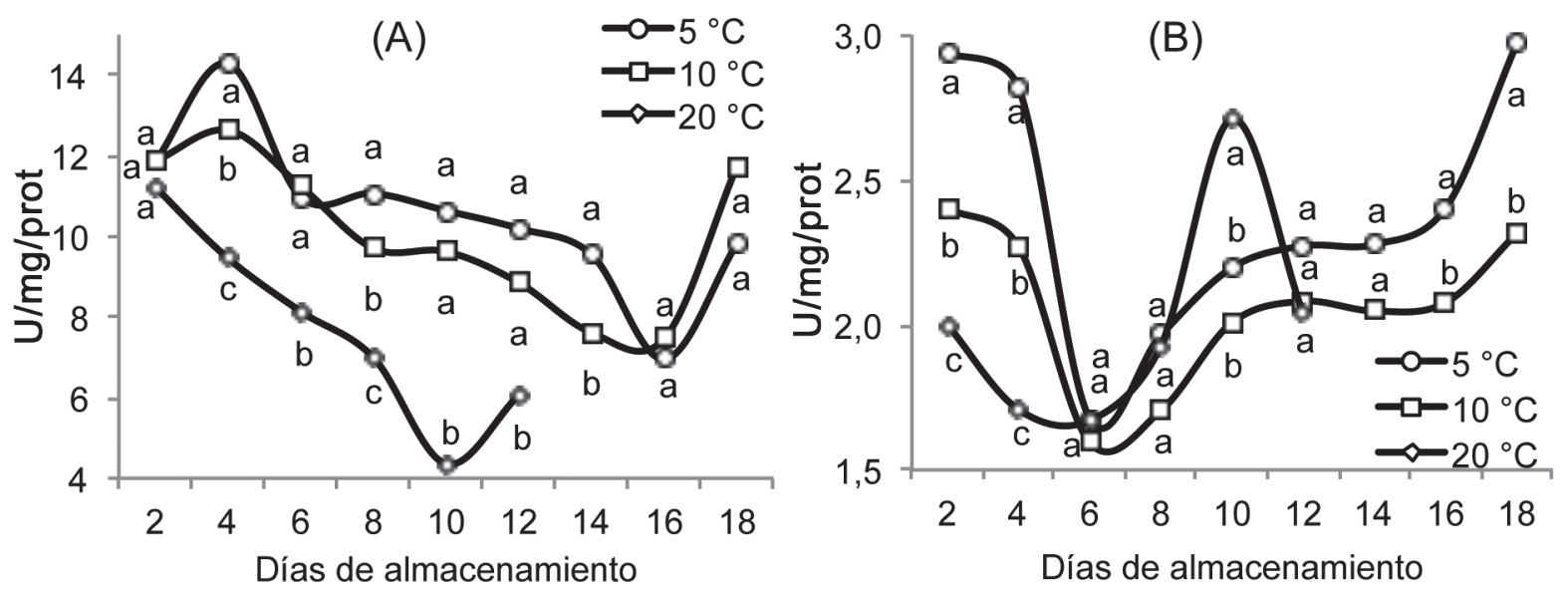

Figura 2. Actividad enzimática de catalasa (A) y superóxido dismutasa (B) en albahaca 'Nufar' almacenada durante dieciocho días a 5, 10 y $20^{\circ} \mathrm{C}$. Texcoco, Estado de México, México. 2012.

Medias con igual letra entre los tratamientos de refrigeración en cada día de almacenamiento no son estadísticamente diferentes de acuerdo con Tukey $(\mathrm{p} \leq 0.05)$.

En este trabajo dicha enzima presentó la mayor actividad a los 2 y 4 DDA en los tratamientos de 5 y 10 ${ }^{\circ} \mathrm{C}(2,9$ y $2,4 \mathrm{U} / \mathrm{mg} /$ prot, respectivamente) (Figura 2), siendo estadísticamente diferentes $(\mathrm{p}<0,05)$ entre sí y con el de $20^{\circ} \mathrm{C}(2,0 \mathrm{U} / \mathrm{mg} /$ prot $)$; de manera semejante, se ha reportado la actividad más alta de la enzima SOD en hojas maduras de nuez vómica (Strychnos nuxvomica) (5,3 U/mg/prot) (Vijayakumar et al., 2009). Posteriormente, esta actividad disminuyó hasta los 6 DDA y a partir de los 8 DDA se incrementó hasta el final del almacenamiento, en donde el tratamiento de $5{ }^{\circ} \mathrm{C}$ presentó los valores más altos de actividad y únicamente en 16 y 18 DDA fue estadísticamente diferente $(\mathrm{p}<0,05)$ al de $10{ }^{\circ} \mathrm{C}$. Los resultados concuerdan con un estudio celular en guisantes (Pisum sativum) donde la actividad de SOD disminuyó con el tiempo, y en la etapa de senescencia la actividad de la SOD mostró un aumento considerable (Palma et al., 2006).

A 10 DDA, los tratamientos de refrigeración fueron estadísticamente diferentes $(\mathrm{p}<0,05)$ al de 20 ${ }^{\circ} \mathrm{C}$, que presentó un incremento en la actividad $(2,7$ $\mathrm{U} / \mathrm{mg} / \mathrm{prot}$ ), igualmente se reportó en extractos de $O$. sanctum un aumento en el nivel de la SOD impidiendo el incremento de los niveles de peroxidación lipídica, lo que atenúa la formación excesiva de especies reactivas de oxígeno (Arivulchelvan et al., 2012). Diversos autores han observado un aumento en la actividad de SOD durante la senescencia; en pera (Pyrus communis) almacenada a $5{ }^{\circ} \mathrm{C}$ a los 28 días de almacenamiento (Ding et al., 2009); en pimiento morrón (Capsicum annuum) almacenado a $5{ }^{\circ} \mathrm{C}$ a los 21 días (Cuadra y Del Amor, 2010); y en manzana (Malus domestica) almacenada a $0{ }^{\circ} \mathrm{C}$, lo cual sugiere un papel de protección en la senescencia contra radicales libres (Du y Bramlage, 1994).

Peroxidasa. Esta enzima se encuentra en la mayoría de los tejidos de la planta y tiene varias funciones relacionadas con la maduración de la fruta, incluyendo la síntesis de la pared celular, los cambios en la plasticidad de la pared celular y lignificación (Zolfaghari et al., 2010). En los primeros días se observó una ligera disminución en la actividad de la peroxidasa (POD), la cual aumentó posteriormente (Figura 3), esto concuerda con lo observado en flores de gerbera (Gerbera jamesonii) 'Richard' y 'Synphonie', en donde detectaron una disminución en la actividad de POD durante los primeros seis días y posteriormente un incremento de esta enzima (Amãriutei et al., 1986).

A 2, 4, 8 y 12 DDA, hubo diferencia estadística $(p<0,05)$ entre los tratamientos de refrigeración con respecto al testigo, quien presentó los mayores valores en la actividad de POD, excepto a los 2 DDA. También en frutos de zapote mamey (Pouteria sapota) almacenados a $20{ }^{\circ} \mathrm{C}$ se observó que la actividad 

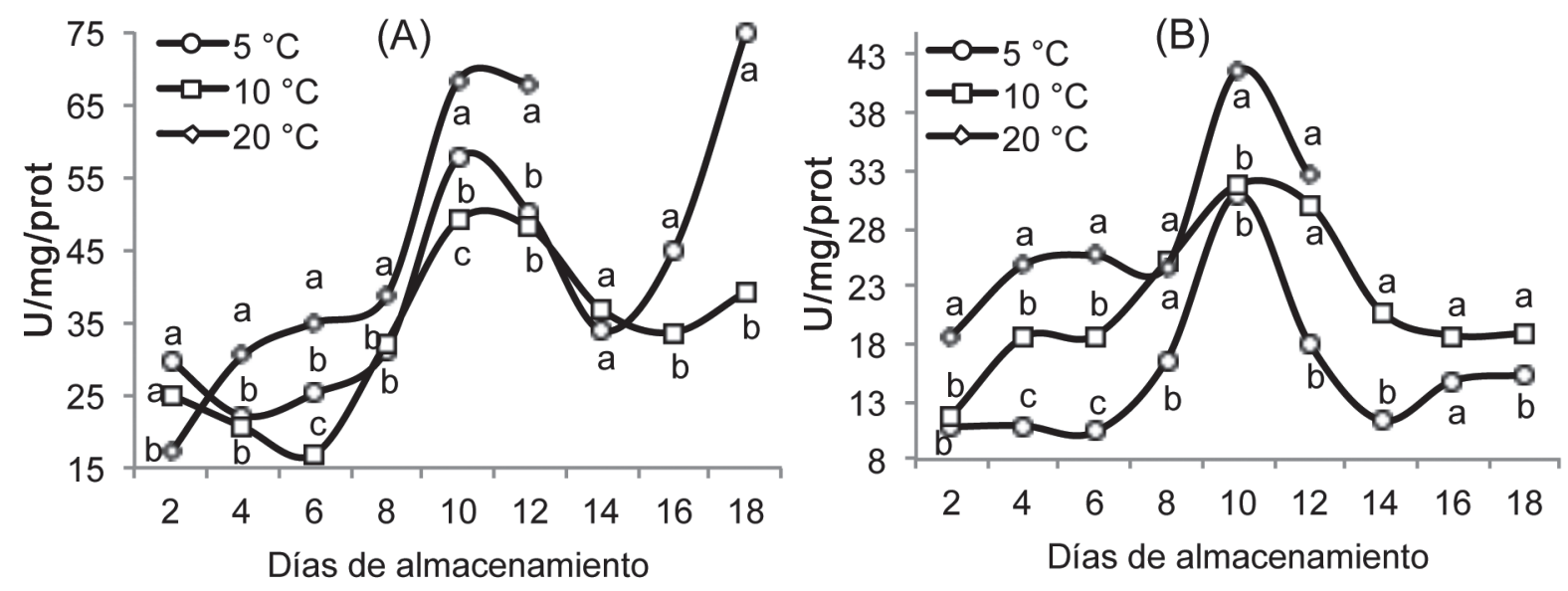

Figura 3. Actividad enzimática de peroxidasa (A) y polifenoloxidasa (B) en albahaca 'Nufar' almacenada durante dieciocho días a 5, 10 y $20^{\circ} \mathrm{C}$. Texcoco, Estado de México, México. 2012.

Medias con igual letra entre los tratamientos de refrigeración en cada día de almacenamiento son estadísticamente iguales de acuerdo con Tukey $(\mathrm{p} \leq 0,05)$.

de POD aumentó durante la maduración (Pérez et al., 1999; Alia et al., 2005). A 6 y 10 DDA, los tres tratamientos fueron estadísticamente diferentes $(\mathrm{p}<0,05)$ entre sí, siendo el de $20{ }^{\circ} \mathrm{C}$ quien a los 10 DDA presentó la mayor actividad $(68,3 \mathrm{U} / \mathrm{mg} / \mathrm{prot})$, de manera semejante la actividad de POD en flores de gerbera (Gerbera jamesonii) presentó una tendencia a incrementar su actividad durante los primeros diez días (Trujillo et al., 2006).

A partir de 12 DDA se observó una disminución en la actividad, y a 16 DDA, aumentó en los tratamientos de refrigeración y fueron estadísticamente diferentes $(\mathrm{p}<0,05)$, siendo el tratamiento de $5{ }^{\circ} \mathrm{C}$ quien presentó los mayores valores ( 45,0 y $74,9 \mathrm{U} / \mathrm{mg} /$ prot). Este patrón observado en la actividad de la POD, concuerda con los resultados en cultivares de kiwi (Actinidia deliciosa) almacenados en frío a $1{ }^{\circ} \mathrm{C}$, en donde la actividad de la POD aumentó inicialmente y luego disminuyó en la semana 18 de almacenamiento (Zolfaghari et al., 2010).

Polifenoloxidasa. Esta enzima se encuentra ampliamente distribuida en las hojas de las plantas y sus cambios durante la maduración, probablemente se relacionan con la protección de la planta contra el estrés biótico y abiótico (Ortega et al., 2010). En relación a la actividad de la polifenoloxidasa (PFO), a 2 y $10 \mathrm{DDA}$ en los tratamientos de refrigeración se observó diferencia estadística $(\mathrm{p}<0,05)$ a una temperatura de $20^{\circ} \mathrm{C}$, la cual reporta los valores más altos de actividad (18,6 y 41,7 U/mg/prot). Resultados similares se encontraron en las hojas y durante la maduración del olivo (Olea europaea), puesto que se observó un incremento significativo y exponencial de la actividad de PFO (Ortega et al., 2010); asimismo en frutos de melocotón (Prunus pérsica) almacenado a $10{ }^{\circ} \mathrm{C}$ también se encontró un aumento gradual en el almacenamiento (Ding et al., 2009).

A los 4 y 6 DDA, hubo diferencia estadística significativa $(\mathrm{p}<0,05)$ entre los tres tratamientos, siendo el de $5{ }^{\circ} \mathrm{C}$, el que presentó los menores valores en estos días (10,9 y 10,4 U/mg/prot); probablemente debido a que la enzima fue estable a temperaturas más bajas (Kavrayan y Aydemir, 2001). De igual forma en frutos de berenjena (Solanum melongena) almacenados a temperatura ambiente, la actividad de PFO aumentó durante el almacenamiento, por el contrario en fruta a $10{ }^{\circ} \mathrm{C}$, la actividad disminuyó durante el almacenamiento (Mishra et al., 2013). A los 8 y 12 DDA, el tratamiento de $20{ }^{\circ} \mathrm{C}$ fue estadísticamente igual con el de $10^{\circ} \mathrm{C}$, pero diferente con el de $5{ }^{\circ} \mathrm{C}(\mathrm{p}<0,05)$, que presentó los menores valores de actividad en dichos días (16,6 y $18,0 \mathrm{U} / \mathrm{mg}$ / prot). Después de 12 DDA, la actividad disminuyó en los tratamientos de refrigeración; similar a lo reportado en un estudio de ocho variedades de albahaca donde se mostraron muy bajos niveles de PFO después de 
un almacenamiento de dos meses de refrigeración (Venere et al., 2002).

El contenido de fenoles totales y capacidad antioxidante, así como la actividad enzimática de peroxidasa y polifenol oxidasa fue afectado negativamente por el frío durante el almacenamiento a $5{ }^{\circ} \mathrm{C}$. Asimismo, a dicha temperatura se incrementó la actividad de catalasa y superóxido dismutasa, aunque fue en decremento en los dieciocho días de almacenamiento. Por lo tanto, se sugiere el almacenamiento a $10{ }^{\circ} \mathrm{C}$ para disminuir la activación de los sistemas enzimáticos oxidativos.

\section{LITERATURA CITADA}

Ahmed, S., y S.B. Hussain. 2009. Ascorbic acid, carotenoids, total phenolic content and antioxidant activity of various genotypes of Brassica oleracea Encephala. J. of Med. and Biol. Sci. 3(1):1-8.

Alia, I., M.T. Colinas, M.T. Martínez, y M.R. Soto. 2005. Daños por frío en zapote mamey (Pouteria sapota (Jacq.) H.E. Moore and Stearn) II: Cambios en fenoles totales y actividad enzimática. Rev. Fitot. Mex. 28:2532.

Amãriutei, A., I. Burzo, y C. Alexe. 1986. Researches concerning some metabolism aspects of cut gerbera flowers. Acta Hortic. 181:331-337.

Aquino B., E.N., y E. Mercado. 2004. Effects of polyphenol oxidase and peroxidase activity, phenolics and lignin content on the browning of cut jicama. Postharvest Biol. Tech. 33:275-283.

Arcila, C.C., G. Loarca, S. Lecona, y E. González. 2004. El orégano: propiedades, composición y actividad biológica de sus componentes. ALAN 54(1):100-111.

Arivuchelvan, A., S. Murugesan, y P. Mekala. 2012. Antioxidant properties of Ocimum sanctum in broilers treated with high doses of gentamicin. Indian J. Drugs Dis. 1:143-146.

Ayala, J.F., S.Y. Wang, C.Y. Wang, y G.A. González. 2004. Effect of storage temperatures on antioxidant capacity and aroma compounds in strawberry fruit. Food Sci. Technol. 37:687-695.

Balois M. R., M.T. Colinas C.B. Peña, S.H. Chávez, y I. Alia. 2008. Sistema enzimático antisenescencia catalasa-superóxido dismutasa, de frutos de pitahaya (Hylocereus undatus) almacenados con frío. Rev. Chapingo Serie Horticultura 14:295-299.
Beyer, F.W., e I. Fridovich. 1987. Assaying for superoxide dismutase activity: some large consequences of minor changes in conditions. Anal. Biochem. 161:559-566.

Blackwell, R.D., A.J.S. Murray y P.J. Lea. 1990. Enzymes of photorespiratory carbon pathway. En: P.J. Lea, editor, Methods in plant biochemistry. Academic Press, USA. p. 129-144.

Bradford, M.M. 1976. A rapid and sensitive method for the quantitation of microgram quantities of protein utilizing the principle of protein-dye binding. Anal. Biochem. 72:248-254.

Cantwell, M.I., G. Peiser, y E. Mercado. 2002. Induction of chilling injury in jicama (Pachyrhizus erosus) roots: changes in texture, color and phenolics. Postharvest Biol. Tec. 25:311-320.

Cantwell, M.I., y M.S. Reid. 2007. Sistemas de manejo postcosecha: hierbas frescas. En: A. Kader, editor, Tecnología postcosecha de cultivos hortofrutícolas. Series de Horticultura Poscosecha No. 24. University of California, Davis, USA. p. 367-372.

Connor, A.M., J.J. Luby, J.F. Hancock, S. Berkheimer, y E.J. Hanson. 2002. Changes in fruit antioxidant activity among blueberry cultivars during cold temperature storage. J. Agric. Food Chem. 50:893-898.

Cuadra-Crespo, P., y F.M. Del Amor. 2010. Effects of postharvest treatments on fruit quality of sweet pepper at low temperature. J. Sci. Food Agric. 90:2716-2722.

Ding, Z., S. Tian, X. Meng, y Y. Xu. 2009. Hydrogen peroxide is correlated with browning in peach fruit stored at low temperature. Front. Chem. Eng. China 3:363-374.

Du, Z., y W.J. Bramlage. 1994. Superoxide dismutase activities in senescing apple fruit (Malus domestica Borkh.). J. Food Sci. 59:581-584.

Flurkey, W.H., y J. Jen. 1978. Peroxidase and polyphenoloxidase activities in developing peaches. $\mathrm{J}$. Food Sci. 43:1828-1831.

García, T. S., B. De Pascual Santos, y G.J.C. Rivas. 2004. Evaluation of the antioxidant properties of fruits. Food Chem. 84:13-18.

Ghasemnezhad, M., M. Sherafati y G.A. Payvast. 2011. Variation in phenolic compounds, ascorbic acid and antioxidant activity of five coloured bell pepper (Capsicum annuum) fruits at two different harvest times. J. Functional Foods 3:44-49.

Giannopolities, C. N. y S.K. Ries. 1977. Superoxide dismutases. Plant Physiol. 59:309-314. 
Hammer, E.F. 1993. Oxidoreductases. En: T. Nagodawhitana, editor, Enzymes in food processing. Academic Press, USA. p. 221-247.

Hussain, A.I., F. Anwar, S.T. Hussain, y R. Przybylski. 2008. Chemical composition, antioxidant and antimicrobial activities of basil (Ocimum basilicum) essential oils depends on seasonal variations. Food Chem. 108:986995.

Javanmardi, J., C. Stushnoffb, E. Lockeb, y J.M. Vivancob. 2003. Antioxidant activity and total phenolic content of Iranian Ocimum accessions. Food Chem. 83:547-550.

Jayasinghe, Ch., N. Gotoh, T. Aoki, y S. Wada. 2003. Phenolics Composition and Antioxidant Activity of Sweet Basil (Ocimum basilicum L.) J. Agric. Food Chem. 51:4442-4449.

Jin, P., S.Y. Wang, Ch. Y. Wang, y Y. Zheng. 2011. Effect of cultural system and storage temperature on antioxidant capacity and phenolic compounds in strawberries. Food Chem. 124:262-270.

Juliani, H.R., y J. E. Simon. 2002. Antioxidant activity of Basil. En: J. Janick, y A. Whipkey, editores, Trends in new crops and new uses. ASHS Press, Alexandria, VA. p. 575-579.

Kalt, W. 2005. Effects of production and processing factors on mayor fruit and vegetable antioxidants. J. Food Sci. 70:11-19.

Kaur, C, y H.C. Kapoor. 2001. Antioxidants in fruits and vegetables: the millennium's health. Int. J. Food Sci. Tech. 36:703-725.

Kavrayan, D., y T. Aydemir. 2001. Partial purification and characterization of polyphenoloxidase from peppermint (Mentha piperita). Food Chem. 74:147154.

Kevers, C., M. Falkowski, J. Tabart, J.O. Defraigne, J. Dommes, y J. Pincemal. 2007. Evolution of antioxidant capacity during storage of selected fruits and vegetables. J. Agric. Food Chem. 55:8596-8603.

Kirca, A., y E. Arslan. 2008. Antioxidant capacity and total phenolic content of selected plants from Turkey. Int. J. Food Sci. Tech. 43:2038-2046.

Kliebenstein, D.J., R.A. Monde, y R.L. Last. 1998. Superoxide dismutase in Arabidopsis: an eclectic enzyme family with disparate regulation and protein localization. Plant Physiol. 118:637-650.

Kuskoski, E.M., A.G. Asuero, A.M. Troncoso, J. Mancini F., y R. Fett. 2005. Aplicación de diversos métodos químicos para determinar actividad antioxidante en pulpa de frutos. Ciênc. Tecnol. Aliment. 25:726-732.
Kwee, E. M. y E.D. Niemeyer. 2011. Variations in phenolic composition and antioxidant properties among 15 basil (Ocimum basilicum L.) cultivars. Food Chem. 128:1044-1050.

Laminkanra, O. 1995. Enzymatic browning of muscadine grapes products. En: C.L. Lee, y J.R. Whitaker, editores, Enzymatic browning and its prevention. ACS. Washington DC., USA. p. 166-177.

Lange, D.D., y A.C. Cameron. 1994. Postharvest shelf life of sweet Basil (Ocimum basilicum). HortSci. 29: 102-103.

Liang, Y., F. Hu, M. Yang, y J. Yu. 2003. Antioxidative defenses and water deficit-induced oxidative damage in rice (Oryza sativa L.) growing on non-flooded paddy soils with ground mulching. Plant Soil 257(2): 407-416.

Martínez, J., y R. Muñoz. 2001. Characterization of Betacyanin Oxidation Catalized by a Peroxidase from Beta vulgaris L. Roots. J. Agric. Food Chem. 49:40644068.

Mishra, B.B., G. Gautam, y A. Sharma. 2013. Free phenolics and polyphenol oxidase (PPO): The factors affecting post-cut browning in eggplant (Solanum melongena). Food Chem. 139:105-114.

Núñez-López, V., M.T. Martínez-Damián, y M.T. ColinasLeón. 2012. Fisiología poscosecha de albahaca (Ocimum basilicum L.) con y sin acolchado. Rev. Chapingo Serie Horticultura 18:307-315.

Oboh, A. 2004. Change in ascorbic acid, total phenol and antioxidant activity of sun dried commonly consumed green leafy vegetables in Nigeria. Nutr. Health. 18: 29-36.

Ortega-García, F., S. Blanco, M.A. Peinado, y J. Peragón. 2010. Polyphenol oxidase and Oleuropein in Olives and their changes during olive ripening. En: V.R. Preedy, y R.R. Watson, editores, Olives and olive oil in health and disease prevention. Academic Press, USA. p. 233-238.

Oueslati, S., B.N. Karray, H. Attia, M. Rabhi, R. Ksouri, y M. Lachaal. 2010. Physiological and antioxidant responses of Mentha pulegium (Pennyroyal) to salt stress. Acta Physiol. Plant. 32:289-296.

Ozgen, M, N.R. Reese, Z.A. Tulio, C.J. Scheerens, y R.A. Miller. 2006. Modified 2,2-Azino-bis-3ethylbenzothiazoline-6-sulfonic acid (ABTS) method to measure antioxidant capacity of selected small fruits and comparison to ferric reducing antioxidant power (FRAP) and 2,2'-diphenil-1-picrylhydrazyl (DPPH) methods. J. Agric. Food Chem. 54:1151-1157. 
Palma, J.M., A. Jiménez, L.M. Sandalio, F.J. Corpas, M. Lundqvist, M. Gómez, F. Sevilla, y L.A. Del Río. 2006. Antioxidative enzymes from chloroplasts, mitochondria, and peroxisomes during leaf senescence of nodulated pea plants. J. Exp. Bot. 57:1747-1758.

Pathare, P. B., O.U. Linus, y A.S.F. Al-Julanda. 2013. Colour measurement and analysis in fresh and processed foods: a review. Food Bioprocess Technol. 6:36-60.

Pérez, T.G., O.I. Vargas, J.C. Díaz, y M.A. Téllez. 1999. Actividad de polifenoloxidasa y peroxidasa en frutos de mamey sapote (Pouteria sapota). Rev. Iberoam. Tecnol. Postcosecha 1:120-125.

Prior, R.L., y G. Cao. 2000. Antioxidant phytochemicals in fruits and vegetables: diet and health implications. HortSci. 35:588-592.

Rice, A.C., N.J. Miller, y G. Paganga. 1997. Antioxidant properties of phenolic compounds. Trends Plant Sci. 2:152-158.

Rodríguez, J.L., O. Valdés y A. Alemán. 2006. Evaluación de la actividad antioxidante de cinco hierbas aromáticas. Ciencia y Tecnología de los Alimentos 16:30-36.

Sala, J.M. y M.T. Lafuente. 2000. Catalasa enzyme activity is related to tolerance of mandarin fruits to chilling. Postharvest Biol. Tech. 20:81-89.

Salveit, M.E. y L.E. Morris. 1990. Overview on chilling injury of horticultural crops. En: C.Y. Wang, editor, Chilling injury of horticultural crops. CRC Press, Boca Raton, Florida, USA. p. 3-15.

Santos B., C. y A. Scalbert. 2000. Proanthocyanidins and tannin- like compounds: nature, occurrence, dietary intake and effects on nutrition and health. J. Sci. Food Agric. 80:1094-1117.

SAS. 2002. SAS/STAT users guide: Statics, Ver. 9.00. SAS Institute Inc. Cary, North Caroline, USA.

Shahidi, F., P.K. Janitha, y P.D. Wanasundara. 1992. Phenolic Antioxidants. Crit. Rev. Food Sci. Nutr. 32:67-103.

Sharma, R., D. Kaur, D. Oberoi, y D.S. Sogi. 2008. Thermal degradation kinetics of pigments and visual color in watermelon juice. Int. J. Food Prop. 11:439-449.
Soto G., A., G. Ettiene, E. Pérez, L. Sandoval, L. Montilla, y E. Soto. 2012. Propagación y fertilización del cultivo del guanábano. II: Características químicas de frutos. Rev. Fac. Agron. (LUZ) (29):20-36.

Stauffer, C.E. 1989. Enzyme assays for food scientists. Van Nostrand Reinhold, NY, USA.

Stewart R.J., J.B. Sawyer B., C.S. Bucheli, y S.P. Robinson. 2001. Polyphenol Oxidase is Induced by Chilling and Wounding in Pineapple. Aust. J. Plant. Physiol. 28:181-191.

Tabassum, I., Z.N. Siddiqui, y S.J. Rizvi. 2009. Protective effect of Ocimum sanctum on lipid peroxidation, nucleic acids and protein against restraint stress in male albino rats. Biol. Med. 1:42-53.

Trujillo, B.A., H.A. Zavaleta, M.E. Mora, y H.A. López. 2006. Efecto del $\mathrm{CaCl}_{2}$ sobre la actividad enzimática antioxidante durante la vida florero de gerbera (Gerbera jamesonni H. Bolux Ex Hook F.). Rev. Chapingo Serie Horticultura 12:203-209.

Venere, D.D., L. Sergio, V. Linsalata, y G. De Mastro. 2002. Biochemical assessment of basil (Ocimum basilicum L.) cultivars for processing. Acta Hort. 576:177-179.

Vijayakumar, R., C.X. Zhao, R. Gopal, y C.A. Jaleel. 2009. Non-enzymatic and enzymatic antioxidant variations in tender and mature leaves of Strychnos nux-vomica L. (Family: Loganiaceae). C. R. Biologies 332:52-57.

Wang, H., G. Cao, y R.L. Prior. 1996. Total antioxidant capacity of fruits. J. Agric. Food Chem. 44:701-705.

Waterman, P.G., y S. Mole. 1994. Analysis of phenolic plant metabolites. Blackwell Scientific Publications, Oxford, UK.

Zheng, W., y S.Y. Wang. 2001. Antioxidant activity and phenolic compounds in selected herbs. J. Agric. Food Chem. 49:5165-5170.

Zolfaghari, M., M.A. Sahari, M. Barzegar, y H. Samadloiy. 2010. Physicochemical and enzymatic properties of five kiwifruit cultivars during cold storage. Food Bioprocess Technol. 3:239-246. 
